\title{
Organisation de la sécurité des réseaux de distribution d'eau potable
}

\author{
Michel Mesny \\ Ingénieur en chef du génie rural, des eaux et des forêts \\ Ministère de l'Environnement - 14, bd du Général Leclerc \\ 92524 Neuilly-sur-Seine Cedex - Tél. 40818595
}

\section{Introduction}

Habitants des villes, nous sommes entièrement dépendants de réseaux que l'on peut qualifier de stratégiques car leur fonctionnement est indispensable pour nous permettre de vivre et de travailler.

Nous avons besoin de circuits de produits alimentaires, de réseaux d'eau potable pour la boisson, l'hygiène corporelle et les besoins domestiques (cuisine et lavage), de réseaux et circuits d'élimination des déchets (eaux usées et ordures ménagères), de réseaux d'énergie pour nous éclairer, nous chauffer, faire fonctionner nos machines et robots et nous transporter, de réseaux de télécommunications pour nous informer, nous gérer et nous parler. Dans les villes il n'y a guère que l'air qui, bien que souvent pollué, nous soit donné naturellement. Ces réseaux, eau potable, eaux usées, électricité, télécommunications, sont des réseaux de proximité qui viennent jusqu'à nous, dans nos appartements. Plus grande est la ville, plus ces réseaux sont sophistiqués, étendus, obligés d'aller s'alimenter de plus en plus loin, ce qui les rend vulnérables aux accidents et aux agressions, alors que le monde dans lequel nous vivons devient de plus en plus un monde à risques.

Les réseaux de distribution d'eau potable sont particulièrement essentiels et vulnérables :

- une ville sans eau devient vite invivable et dangereuse ; - une contamination de l'eau est relativement facile, détectable souvent par ses seuls effets sur les consommateurs et peut avoir des conséquences meurtrières.

\section{Le contexte technique de la distribution d'eau pota- ble}

La fabrication d'eau potable à partir d'eaux brutes naturelles nécessite en général un traitement, fonction de la qualité de l'eau brute. Certaines caractéristiques minimales sont exigées des eaux brutes pour qu'elles puissent servir à la fabrication d'eau potable et l'eau potable doit satis- faire à des normes de qualité. Pour l'ensemble de ces valeurs limites, existent des normes européennes.

Les sources et les eaux souterraines sont traditionnellement les ressources en eau privilégiées pour l'eau potable, car mieux à l'abri des pollutions que les eaux superficielles. Cependant la montée des nitrates et des pesticides dans les eaux souterraines et l'amélioration des techniques de traitement peuvent conduire à préférer les eaux superficielles, les eaux courantes répondant plus vite aux efforts de dépollution. Systématiquement les eaux de surface sont traitées ; à signaler cependant qu'une ville comme New York est alimentée en eau potable à partir de retenues par simple chloration sans traitement.

Le traitement de l'eau brute nécessite de l'énergie électrique et des produits chimiques. Pour garantir la potabilité de l'eau à l'aval de la station de traitement dans le réseau de distribution, une chloration de l'eau potable est nécessaire, le chlore ayant un pouvoir rémanent de désinfection de l'eau que n'a pas l'ozone.

Un réseau d'eau potable comprend en général des points de prélèvements avec pompage (forages, prises d'eau en rivière), une station de traitement et un réseau de distribution en tuyaux sous pression avec des réservoirs de régulation en général à surface libre. Sans électricté, après vidange des réservoirs surélevés, l'eau s'arrête de couler, sauf cas exceptionnel de réseau gravitaire.

En ce qui concerne plus précisément la France, nous avons 13500 unités de distribution d'eau potable (soit un ratio de 4000 habitants par unité, contre 10000 en Allemagne, 28000 en Suède et de l'ordre de 1000000 en Belgique et en Grande-Bretagne). Cette relative dispersion est compensée par l'existence de grandes sociétés privées d'importance nationale qui interviennent dans la distribution de l'eau potable pour le compte des collectivités locales et desservent ainsi la majorité de la population française. Nous consommons en moyenne 250 litres par jour et par habitant et notre eau provient à $40 \%$ de rivières et lacs, $40 \%$ d'eaux souterraines et $20 \%$ de sources. Nous nous distinguons par une forte consommation d'eaux minérales (4 milliards de litres par an, soit 0,20 litre par jour et par habitant). 


\section{Les dangers}

La nature des dangers est diverse :

- risques technologiques et naturels ;

- négligence ;

- malveillance. Cette dernière catégorie comprend le vandalisme, le terrorisme et les actes de guerre.

Les applications de ces dangers à l'eau potable s'appellent :

- sécheresse, la quantité de la ressource diminue et apparaissent des problèmes de qualité ;

- pollutions accidentelles de la ressource, provenant d'accidents dans l'industrie ou dans le transport des matières dangereuses ;

- interruption de la fourniture d'électricité, l'eau s'arrête de couler au robinet;

- contamination de l'eau potable ;

- destruction d'infrastructures (pompes, station de traitement, tuyaux, réservoirs, circuits électriques, circuits de mesure, de contrôle et de gestion). C'est ainsi qu'en Grande-Bretagne les stations de pompage ont des toits fusibles de façon à limiter les dégâts en cas d'attentats à l'explosif.

Un danger dont on commence à parler dans le milieu civil est l'impulsion électromagnétique nucléaire haute altitude (IEMN-HA ou IEM). Ce phénomène peut être provoqué par l'explosion d'une bombe atomique classique à haute altitude (par exemple $100 \mathrm{KT}$ ou 6 fois la puissance de la bombe d'Hiroshima à $100 \mathrm{~km}$ d'altitude). Une grande superficie comme la France peut alors être arrosée de rayonnements en particulier gamma, pour lesquels tout conducteur non protégé devient antenne et risque d'être traversé et détruit par un courant de grande intensité, sans qu'il y ait d'effets de souffle et de radioactivité sensibles pour la population. La protection contre l'IEM ou durcissement se fait suivant le principe de la cage de Faraday.

Les deux systèmes prioritaires dont la protection contre l'IEM doit être mis en œuvre sont :

- les télécommunications gouvernementales pour le maintien d'une capacité minimale de télécommunications ; - l'alerte et l'information des populations.

Pour les autres secteurs (électricité, informatique, transports routiers, ferroviaires et aériens, alimentation en eau potable, carburants, services de santé et risques technologiques), il est prévu la réalisation d'études de vulnérabilité et de protection, avec propositions éventuelles d'actions en 1993.

Les caractéristiques de l'IEM retenues pour la protection dans le secteur civil correspondent à un champ électrique maximal de 50 kilovolts par mètre avec impulsion unique.

\section{Les plans de secours}

En matière d'eau potable, au niveau départemental les plans ORSEC sont complétés par des plans de secours spécialisés.
La circulaire du ministère de l'intérieur du 27 septembre 1988 demande aux préfets d'élaborer des plans départementaux de secours spécialisés ayant pour objet la lutte contre des perturbations importantes sur un réseau de distribution d'eau potable. Ce sont des plans administratifs de gestion de crise sous l'autorité des préfets. Ils sont certes nécessaires, mais ils ne sont que curatifs et restent donc insuffisants, d'autant plus qu'ils peuvent inciter les exploitants à abandonner leurs responsabilités de service public en cas de crise pour se retourner vers le préfet. En fait l'amélioration de la sécurité de la distribution de l'eau potable nécessite la sécurisation des installations à titre préventif, ce qui ne peut être fait que par les exploitants des réseaux de distribution d'eau potable sous la responsabilité des collectivités maîtres d'ouvrage.

Une évaluation des plans de secours déjà réalisés est en cours de façon à les rendre plus opérationnels, en y associant plus étroitement les gestionnaires des réseaux.

En matière de plans de secours, la démarche à suivre doit être celle adoptée pour les risques industriels. Dans le cadre de la procédure d'autorisation des installations classées pour la protection de l'environnement, le futur exploitant doit réaliser une étude de dangers exposant les risques présentés par l'installation, décrivant les scénarii d'accident et justifiant les mesures prises pour en réduire la probabilité et les effets, ainsi que les moyens d'intervention utilisables en cas de sinistre. Sur la base de cette étude de dangers, l'exploitant établit un plan d'opération interne (P.O.I.) décrivant l'organisation de ses secours et ses procédures d'intervention en cas d'accident. Ce P.O.I. est prolongé par un plan particulier d'intervention (P.P.I.) élaboré par le Préfet et précisant l'organisation des secours en cas d'accident grave débordant l'emprise de l'installation.

Il est certain que celles des collectivités qui assurent en régie le service de l'eau potable et ne peuvent donc faire appel à la solidarité d'une grande société distributrice nationale seront davantage concernées par les plans départementaux.

Ces plans départementaux devront comporter obligatoirement une phase "étude de dangers " et les aspects communication devront y occuper une place importante, car il s'agit bien là d'une responsabilité préfectorale en cas de crise. Ils devront s'appuyer sur les plans de secours locaux (équivalents des plans d'opération interne), que doit élaborer chaque exploitant d'un réseau de distribution d'eau potable, et être harmonisés avec eux de façon à organiser la solidarité départementale. L'informatisation de ces plans de secours départementaux est souhaitable pour les rendre plus efficaces en gestion de crise et plus fiables en réactualisation.

Depuis l'accident PROTEX en 1988, qui a pollué la Loire et a privé d'eau 200000 personnes à Tours pendant une semaine, on admet que, sauf contamination grave, il est préférable de maintenir, après information de la population, la distribution de l'eau, même non potable. 


\section{LA SÉCURITÉ DES RÉSEAUX DE DISTRIBUTION D'EAU POTABLE}

\section{Organisation de la sécurité}

La sécurité du service public d'eau potable est de la responsabilité de la collectivité, maître d'ouvrage, et du gestionnaire à qui elle a pu confier l'exploitation du réseau. Cela suppose à la fois un état d'esprit permanent de vigilance et la mise en place des dispositifs permettant :

- la protection préventive ;

- la détection;

- les solutions de secours.

Les points clefs du réseau doivent avoir été identifiés, leur vulnérabilité appréciée et éventuellement leur classement en point sensible effectué. Les solutions de secours doivent effectivement exister en réserve et non pas être inventées pendant la crise.

\subsection{Les dispositifs de protection}

Les points vulnérables et en particulier les accès à une surface libre d'eau potable (c'est le cas dans les stations de traitement et les réservoirs) doivent être équipés de dispositifs de protection anti-intrusion avec retransmission de l'alarme à un centre de contrôle.

Ces systèmes de protection consistent en :

- clôtures avec détection périmétrale de franchissement ; — portes blindées avec serrures de sécurité et alarmes de contact ;

- capots blindés et verrouillés avec alarmes d'ouverture pour les cheminées d'aération des puits, des captages et des réservoirs.

Comme modèle de protection anti-intrusion, on peut citer en France le réservoir de Montreuil du Syndicat des Eaux d'Ile-de-France (SEDIF).

Par ailleurs, les branchements de distributions doivent être équipés de clapets anti-retour ou mieux de disconnecteurs afin d'éviter toute contamination du réseau à partir d'un point d'utilisation.

En période troublée, une vigilance renforcée doit être mise en place:

- limitation des points d'entrée dans les installations;

- contrôle rigoureux des entrées et des sorties (badges,

filtrage des visiteurs);

- suppression des visites non indispensables;

- surveillance continue de certaines installations par vigiles et chiens ;

- rondes par gendarmerie, police municipale et sociétés de surveillance ;

- éventuellement, surveillance militaire des points classés sensibles.

Ainsi, pendant la crise du Golfe, au début de 1991, les portes des regards d'un aqueduc isolé alimentant Paris ont été soudées par mesure de sécurité.

\subsection{La chloration}

\subsubsection{Lutte contre les contaminations}

Compte tenu de la rémanence du chlore, la chloration est le moyen indispensable pour assurer la désinfection de l'eau dans les réseaux de distribution. En effet, l'ozonation désinfecte l'eau, mais n'a aucun effet rémanent, laissant l'eau potable vulnérable à toute contamination dans le réseau de distribution.

L'objectif de teneur en chlore résiduel dans les réseaux de distribution en France est de $0,05-0,10 \mathrm{mg} / 1$ pouvant être porté à $0,20-0,30 \mathrm{mg} / \mathrm{l}$ en période à risques. Cela demande une chloration de $0,2-0,3 \mathrm{mg} / 1(0,4-0,6 \mathrm{mg} / 1$ en période à risques) à la sortie des usines de traitement des eaux et éventuellement des chlorations additionnelles sur certaines antennes longues des réseaux de distribution.

Des essais et analyses menés par le Centre d'études du Bouchet en collaboration avec la Lyonnaise des eaux Dumez et la Compagnie générale des eaux, au moment de la crise du Golfe, ont montré que la toxine botulinique était neutralisée à partir d'un seuil en chlore résiduel stabilisé à $0,3 \mathrm{mg} / \mathrm{l}$.

Il apparaît ainsi qu'en poussant la chloration à la sortie des usines de traitement et en effectuant des chlorations additionnelles en certains points des réseaux, de façon à maintenir au moins $0,3 \mathrm{mg} / \mathrm{l}$ de chlore résiduel dans le réseau de distribtuion, il serait possible d'assurer la neutralisation d'une possible contamination terroriste par la toxine botulinique.

\subsubsection{Détection des contaminations}

Le chlore résiduel dans le réseau permet également de détecter l'introduction dans le réseau de substances contaminantes, qui consommeraient immanquablement le chlore libre. Un micro capteur " chlore libre " est en cours de mise à point. D'un coût relativement bas, ce capteur aurait une sensibilité de $0,01 \mathrm{mg} / \mathrm{l}$ et pourrait transmettre une alarme en cas de franchissement d'un seuil inférieur. Son installation dans les réseaux de distribution d'eau potable permettrait de constituer un réseau d'alerte contre les contaminations.

\subsubsection{Effets secondaires de la chloration}

L'action du chlore sur les précurseurs (matières organiques et chlorophylle) entraîne la formation de tri-halométhanes, substances suspectées d'être cancérigènes. Cela a conduit les distributeurs d'eau français à abandonner la préchloration des eaux brutes et à ne chlorer qu'en fin de filière de traitement pour limiter la formation de trihalométhanes.

La concentration maximale admissible pour les trihalométhanes est actuellement aux U.S.A. de $100 \mu \mathrm{g} / 1$ (norme EPA). C'est un taux élevé, car certaines villes aux U.S.A., New York en particulier, ne traitent pas leur eau et se contentent de chlorer l'eau brute à $0,7-2 \mathrm{mg} / \mathrm{l}$ de façon à conserver au moins $0,2 \mathrm{mg} / \mathrm{l}$ de chlore résiduel au robinet. Le Conseil des Communautés Européennes n'a pas encore fixé de concentration maximale admissible pour les tri-halométhanes, mais il est important que ne soit pas imposée une norme trop stricte inférieure à $30 \mu \mathrm{g} / \mathrm{l}$, car elle compromettrait la chloration nécessaire pour la sécurité de la distribution de l'eau potable. 


\subsection{La détection des contaminations}

\subsubsection{Analyse des substances toxiques}

Compte tenu du nombre de substances toxiques possibles, il n'est procédé en général à de telles analyses qu'en cas de présomption de contamination (intrusion dans un réservoir par exemple).

\subsubsection{Réaction d'organismes vivants}

L'intérêt des organismes vivants est qu'ils réagissent globalement aux polluants ou contaminants. Le suivi en continu des réactions d'une truite (truitomètre) permet de détecter une pollution des eaux brutes. Cependant la truite peut être moins sensible que l'homme à certains contaminants. L'alerte peut toutefois être donnée de façon plus fine non par la mort de la truite, mais par un comportement anormal enregistré globalement sur une quinzaine de truitelles par effet Doppler et donnant lieu à une pré-alarme.

Le taux de mortalité de certains micro-organismes peut aussi être observé par luminescence, c'est le procédé "MICROTOX ».

Dans tous les cas, la détection à partir d'organismes vivants ne peut être utilisée directement que sur l'eau brute. Sur l'eau traitée, par exemple l'eau potable à la sortie d'un réservoir, il faudrait préalablement déchlorer l'eau dérivée sur le truitomètre ou le microtox.

\subsubsection{Suivie de la teneur en chlore résiduel}

Le chlore résiduel, comme indiqué précédemment, semble constituer le témoin le plus fiable d'une contamination et l'installation des capteurs correspondants est la solution d'avenir.

\subsection{L'alerte}

Lorsque la ressource en eau est une rivière, une station d'alerte située à l'amont de la prise d'eau et analysant en continu certaines caractéristiques de qualité de l'eau permet par transmission automatique des dépassements de seuil de détecter l'arrivée d'une pollution accidentelle et de prendre les mesures nécessaires avant l'arrivée de la pollution au droit de la prise.

D'une façon générale la détection d'une pollution de l'eau brute ou d'une contamination de l'eau potable entraîne une alerte automatique qui, après vérification, doit permettre d'activer un plan d'intervention, soigneusement établi et dosé, pouvant aller, cas extrême, jusqu'à l'interprétation de la distribution.

\subsection{Les solutions de secours}

\subsubsection{Solutions de secours sans interruption de la distribu- tion}

i) Diversification des ressources et maillage et interconnexion des réseaux
La sécurité de l'alimentation en eau potable de la région parisienne apparaît satisfaisante. Il n'en est pas de même dans certaines grandes villes de province, tant en ce qui concerne la ressource que la protection des installations.

$\mathrm{La}$ région parisienne consomme de 2 à 3 millions $\mathrm{m}^{3} /$ jour et est alimentée en eau potable à partir de ressources diverses :

- eaux superficielles (Seine, Marne et Oise) pour $60 \%$, - eaux souterraines (champs captants réalimentés) pour $20 \%$,

- eaux de source en adduction gravitaire pour $20 \%$ du total et $60 \%$ de la seule consommation de Paris.

Ainsi une pollution accidentelle sur deux des trois rivières n'entraîne pas en général de perturbation dans la distribution, par appel renforcé aux autres ressources et éventuellement aux interconnexions entre les divers réseaux. La capacité potentielle d'échange permis par ces interconnexions est de $1900000 \mathrm{~m}^{3} /$ jour.

ii) Réservoirs tampons, permettant une certaine autonomie du réseau en cas de pollution de la ressource

Une réserve correspondant à vingt-quatre heures de consommation est recommandée.

iii) Alimentation électrique de secours par groupes électrogènes, fixes ou mobiles

L'usine d'Orly de la SAGEP (Eau de Paris) a une alimentation de secours par groupes électrogènes fixes (puissance totale : $6000 \mathrm{~kW}$ ), qui est rentabilisée par l'effacement des jours de pointe.

\subsubsection{Solutions de secours en cas d'interruption de la distri- bution normale}

i) Eaux embouteillées

- chez les habitants,

- dans les grandes surfaces.

ii) Eaux ensachées

- Réserves familiales, en Suisse chaque foyer a l'obligation de conserver dix litres d'eau par personne.

- Stocks stratégiques.

- Equipements mobiles d'ensachage avec possibilité d'adjoindre une unité de traitement.

iii) Réseau de distribution partiel, alimenté à partir d'une ressource en eau protégée des contaminations et desservant des bornes-fontaines et certains abonnés prioritaires (hôpitaux...)

Paris pourrait utiliser la nappe profonde de l'albien. Un tel réseau secondaire desservant des bornes-fontaines à partir de sources existe à Zurich, comme nous le verrons plus loin.

iv) Kits individuels de potabilisation de l'eau. 


\section{Exemple de la Suisse}

\subsection{Ordonnance sur la garantie de l'approvisionnement en eau potable en temps de crise}

Cette ordonnance a été promulguée le 20 novembre 1991 par le Conseil fédéral, elle s'applique aux services publics et privés de distribution d'eau potable et vise à garantir l'approvisionnement en eau potable en temps de crise (catastrophe naturelle, accident technologique majeur, sabotage, actes de guerre, y compris l'impulsion électromagnétique nucléaire). Pour cela elle stipule que :

1. Les mesures doivent être prises et les dispositifs de secours exister pour assurer le service minimal suivant :

- pendant les 3 premiers jours, aucune obligation n'est imposée, l'approvisionnement en eau potable étant assurée par les stocks d'eau embouteillée - 10 litres par personne - que doit détenir chaque foyer ;

- les $4^{\mathrm{e}}$ et $5^{\mathrm{e}}$ jour, 4 litres par personne et par jour ;

- à partir du $6^{\mathrm{e}}$ jour, 15 litres par personne et par jour.

Les niveaux exigés de service minimal seront assurés par la mise à disposition de la population d'eau ensachée, ou par la mise en service d'un réseau secondaire de bornesfontaines (dans ces deux cas, la population devra « aller chercher l'eau »), ou par la mise en œuvre en service partiel du réseau principal à partir d'ouvrages protégés.

2. Tout exploitant de réseau d'eau potable est tenu de soumettre à l'approbation du canton un plan global comportant notamment :

- l'étude des dangers et les scénarii des dégâts possibles;

- les dispositifs permettant de garantir le service minimal ci-dessous ;

- les mesures pour la remise en état des installations.

\subsection{Ville de Zurich}

\subsubsection{Risques couverts}

i) Impulsion électromagnétique

La ville de Zurich est sans doute la seule ville au monde oủ le risque impulsion électromagnétique nucléaire, dont il a été parlé précédemment, a été pris en compte.
La station de pompage principale de la ville, qui abrite également le centre de télécommande et possède ses propres groupes électrogènes, est dans un bâtiment construit pour constituer une cage de Faraday.

Les autres villes, en particulier Genève, s'oriente vers une solution moins onéreuse, du type fusible ou protection localisée pour protéger les installations essentielles et permettre une remise en état rapide.

\section{ii) Contamination nucléaire}

Tous les réservoirs, stations de traitement et puits de forage sont équipés de dispositifs de filtration d'air et de sas avec portes blindées.

\section{iii) Alimentation électrique}

Une alimentation électrique de secours est en place dans les stations principales sous forme de groupes électrogènes fixes et pour les petites stations sous forme de groupes mobiles avec des camions tracteurs protégés contre l'impulsion électromagnétique nucléaire.

\subsubsection{Conception du réseau principal}

Le réseau principal est sécurisé par l'existence d'une triple boucle, hydraulique, électrique et de télécommande.

\subsubsection{Solutions de secours}

Si le réseau principal est fortement perturbé, peut être mis en service un réseau secondaire alimenté à partir de ressources en eau protégées, constituées par des sources, et desservant un réseau de bornes-fontaines. Il en existe une centaine, utilisées comme fontaines en temps normal pour l'agrément de la ville. En situation de crise, sur chaque fontaine peut être branché un dispositif de chèvre comportant 36 robinets, permettant ainsi 3600 points de distribution d'eau pour une population de 350000 habitants, soit environ un point de distribution d'eau pour 100 habitants.

Si ce réseau secondaire est également hors service, des unités mobiles de fabrication sont capables de débiter chacune par heure 1200 sachets d'un litre d'eau pour distribution à la population.

\section{Conclusions}

L'alerte de la crise du Golfe au début de 1991 a montré la capacité de mobilisation des distributeurs d'eau potable aux problèmes de sécurité. Cependant la sécurité d'un réseau d'eau potable ne s'improvise pas, l'équipement des réseaux doit intégrer cet objectif de sécurisation, qui doit se concrétiser par une obligation de résultat, c'est-à-dire de niveau de service minimal en cas de crise.

Il appartient aux collectivités et aux gestionnaires de réseaux de choisir les moyens optimaux pour assurer en toute circonstance le niveau de service nécessaire. Nous sommes entrés dans une civilisation à risques et l'eau est au cœur de tous les dangers. 


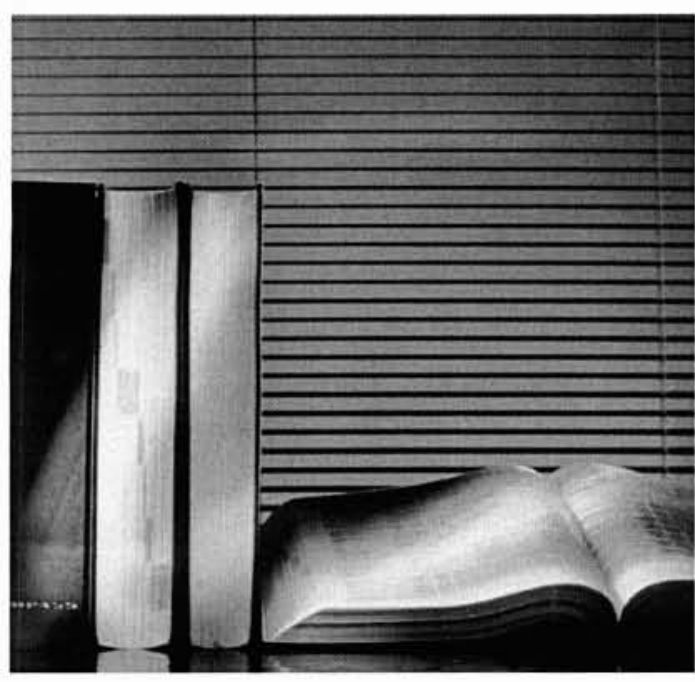

LE
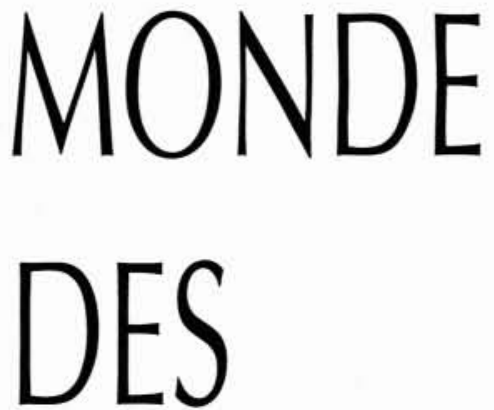

ENTREPRISES

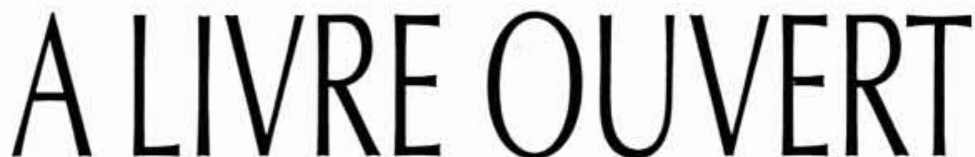

Quels que soient vos marchés, vos ambitions ou vos besoins, les produits de Kompass Edition vous permettent d'accroître, de diversifier, d'affermir vos relations d'affaires, simplement et efficacement.

- Kompass France,

- Kompass Région,

- Kompass Professionnel,

- Kompass International,

en une série d'ouvrages professionnels et thématiques, Kompass décrit avec rigueur et exhaustivité le monde des entreprises.

Coordonnées, décideurs, chiffre d'affaires, effectif, produits, marques... avec les annuaires Kompass, vous disposez d'une source d'informations de qualité, dont la fiabilité reconnue vous assurera le succès.
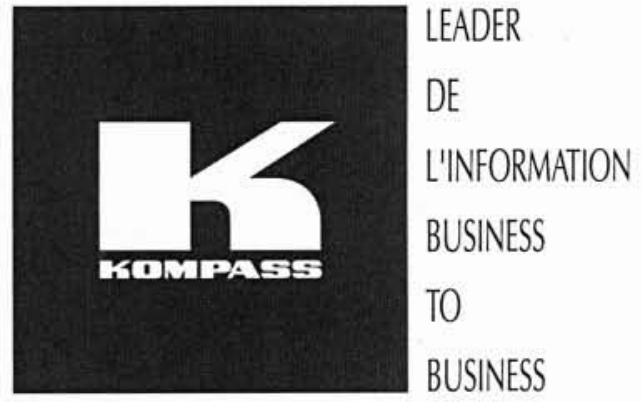

$\mathrm{N}$ 'hésitez pas à vous renseigner au :

(1) 43593759 ,

ou écrivez à

Kompass France, 22, av. F. D. Roosevelt - 75008 PARIS.

Fax : (1) 45638349. 